\title{
Optical Distortion
}

National Cancer Institute

\section{Source}

National Cancer Institute. Optical Distortion. NCI Thesaurus. Code C62889.

Problem associated with an optical defect in an image-forming system whereby the image is not the shape of an ideal image of the object. 